\title{
Crystal Growth and Spectroscopic Performance of Large Crystalline Boules of $\mathrm{CsCaI}_{3}: \mathrm{Eu}$ Scintillator
}

\author{
A Lindsey*, W. McAlexander, L Stand, Y. Wu, M. Zhuravleva, and C. L. Melcher \\ Scintillation Materials Research Center - Materials Science and Engineering Department - The University \\ of Tennessee - Knoxville TN, USA \\ *Corresponding author Tel: 423943 9508. E-mail: AColemanLindsey@ gmail.com
}

\section{Abstract}

The crystal growth of $\mathrm{CsCaI}_{3}$ :Eu with improved quality over prior efforts for use in X-ray and gamma-ray detection applications is reported. Boules were grown using the vertical Bridgman technique in quartz ampoules at 15,22 , and $35 \mathrm{~mm}$ diameters. $\mathrm{CsCaI}_{3}: \mathrm{Eu}$ crystals show evidence of a heavily twinned microstructure likely caused by a solid-solid phase transformation of the crystal during the cooling process. Energy resolution (FWHM) in large and small samples taken from crystalline boules vary between $13 \%$ and $4.5 \%$ for $662 \mathrm{KeV}$ gamma rays indicating volumetric non-uniformity. Scintillation decay time is shown to increase with volume. Proportionality of the light yield of $\mathrm{CsCaI}_{3}: \mathrm{Eu}$ is reported for the first time.

\section{Keywords}

Bridgman technique, Halides, Calcium compounds, Scintillator materials, Scintillators

\section{Introduction}

Improvements to the capabilities and cost of radiation detection and radioactive isotope identification devices are an integral component to the increasing needs of national security operations and the medical imaging industry. The evolving geopolitical climate requires efforts to enforce nuclear non-proliferation agreements as well as enhance domestic security to prevent the movement and trade of illegal radiological contraband across and within borders. Despite the unsurpassed performance in radionuclide identification, the cost associated with production and operation of semiconductor based radiation detectors such as high purity germanium (HPGe) and cadmium telluride prohibits their widespread use and availability. As a result, research and development into detector technology based on new scintillator materials with improved spectroscopic performance, namely light yield and energy resolution, is required to advance this capability [1].

Single crystal metal halide scintillators offer superior light yield and detection efficiency over organics, liquids, and many oxide based scintillators. One drawback is the difficulty encountered when attempting to scale up the growth process to larger diameters for economical detector fabrication. Single crystal synthesis using melt growth techniques are more readily scalable over vapor deposition or solution growth methods to meet the demand for industry. Adapting growth processes developed at the laboratory scale to an industrial scale is a fundamental challenge to development of new metal halide scintillator materials. Over the last decade, a few binary metal 
halide materials with energy resolution of $\sim 3 \%$ (FWHM) at $662 \mathrm{KeV}$ have been commercialized such as $\mathrm{SrI}_{2}: \mathrm{Eu}, \mathrm{LaBr}_{3}: \mathrm{Ce}$ and $\mathrm{CeBr}_{3}$, [2, 3]. However, the cost of these new generation of materials remains prohibitively high due to the low yield of high quality crystals that is in part due to growth processes which remain difficult to scale up to larger diameters. Obstacles may include large anisotropy in thermal expansion which requires control over the orientation of the growing crystal. Additionally, coupled with the typically low mechanical strength of metal halides, large single crystals are prone to fracture during growth and cooling rendering them unusable. Consequently, these effects have caused the deployment of these next generation materials to be limited.

In recent years, the search for new ternary compositions with similar performance to the aforementioned binary compositions have yielded many new materials which may offer reduced cost of development with improved performance over current technology utilizing NaI:Tl [4, 5]. Each new ternary composition poses unique advantages and disadvantages to their development such as activator distribution, optical quality, and mechanical strength. Moreover, compositions possessing promising performance at the laboratory scale $\left(<1 \mathrm{~cm}^{3}\right)$ must be assessed in terms of their feasibility for scale up to larger diameter growth processes. This is largely assessed by volumetric uniformity in scintillation performance, and a resistance to fracture. Complicating this effort is the hygroscopic nature of nearly all of the metal halides requiring special care to be taken in the preparation, handling, and investigation of these materials.

Research into new ternary compositions with promising scintillation performance has accelerated in the last decade. For instance, melt grown crystals of $\mathrm{CsCaBr}_{3}: \mathrm{Eu}$ are reported possess a light yield of $\sim 29,000$ photons/MeV and 9.3\% energy resolution at $662 \mathrm{KeV}$ [6]. Recent reports on the scintillation properties of $\mathrm{CsCaI}_{3} \mathrm{Eu}(\mathrm{CCI})$ crystals grown from the melt using the vertical Bridgman technique (VBT) have shown promising performance with $\sim 40,000$ photons/MeV yet cite poor crystalline quality and inhomogeneity as causes of decreased energy resolution [7, 8]. This has prompted an effort to produce higher quality crystals of CCI through growth experiments aimed at identifying and obviating commonly encountered bulk defects using VBT. In this manner we hope to assess the potential performance of CCI and evaluate the advantages and disadvantages to its further development.

In consideration for spectroscopic applications, reproducible detector volumes approaching 1 cubic inch represents a milestone for development. In this present work, we report the results of growth experiments using VBT in producing boules of CCI at 15, 22, and $35 \mathrm{~mm}$ diameters as well as their handling. The scintillation performance including light yield, energy resolution, and decay time for various volumes of CCI is shown. The proportionality of CCI is also reported for the first time.

\section{Experimental Method and Equipment}




\subsection{Crystal Growth}

75 Due to the hygroscopic nature of the precursor starting materials, all handling took place in a nitrogen flushed MBraun glove box maintaining <0.1 ppm moisture and oxygen to prevent deliquescence and or contamination prior to synthesis. Precursor materials consisting of anhydrous CsI, $\mathrm{CaI}_{2}$, and $\mathrm{EuI}_{2}$ with $99.99 \%$ or better purity in beaded form were used for the synthesis of $\mathrm{CsCaI}_{3}$. A europium concentration of $3 \mathrm{~mol} \%$ was attained through substituting $\mathrm{EuI}_{2}$ for $\mathrm{CaI}_{2}$ in an otherwise 1:1 mixture of $\mathrm{CsI}: \mathrm{CaI}_{2}$. This activator concentration was previously determined to produce optimal results in the CCI matrix [8]. Quartz growth ampoules were loaded with the precursor components within the controlled atmosphere and then transported to a high vacuum furnace and dried at $250^{\circ} \mathrm{C}$ and $10^{-7}$ torr for several hours prior to sealing with an $\mathrm{H}_{2}-\mathrm{O}_{2}$ torch. The vacuum sealed ampoules containing the unreacted charges were suspended into the growth furnace by a quartz hook attached at the top of the ampoule and connected to the translation mechanism. The charges were then heated to $\sim 800^{\circ} \mathrm{C}$ for 24 hours in order to allow all the material to melt and mix thoroughly prior to beginning growth.

Growth experiments were performed in a custom built two zone transparent vertical Bridgman furnace and recorded with continuous observation of the growth process using time lapse photography. A more detailed description of the equipment can be found in [9]. Using this experimental setup, the crystal appearance along with the shape of the growth interface could be observed at all times. This is especially useful for growth of materials which exhibit a large supercooling behavior that naturally make the self-seeding process more challenging to control.

Differential scanning calorimetry (DSC) measurements of CCI are shown in Figure 1. The melt of CCI exhibits a characteristic supercooling of $\Delta \mathrm{T} \approx 35^{\circ} \mathrm{C}$ in the absence of any solidified portion. As a result of this, ampoules with a capillary section at the bottom were used to control the initial formation of a randomly oriented seed crystal. A melting point of $686^{\circ} \mathrm{C}$ is used to prevent remelting of the self-seed. Evidence of a suspected phase transition occurring at $\approx 255^{\circ} \mathrm{C}$ can also be detected in the DSC heating and cooling curves (Inset of Figure 1). 


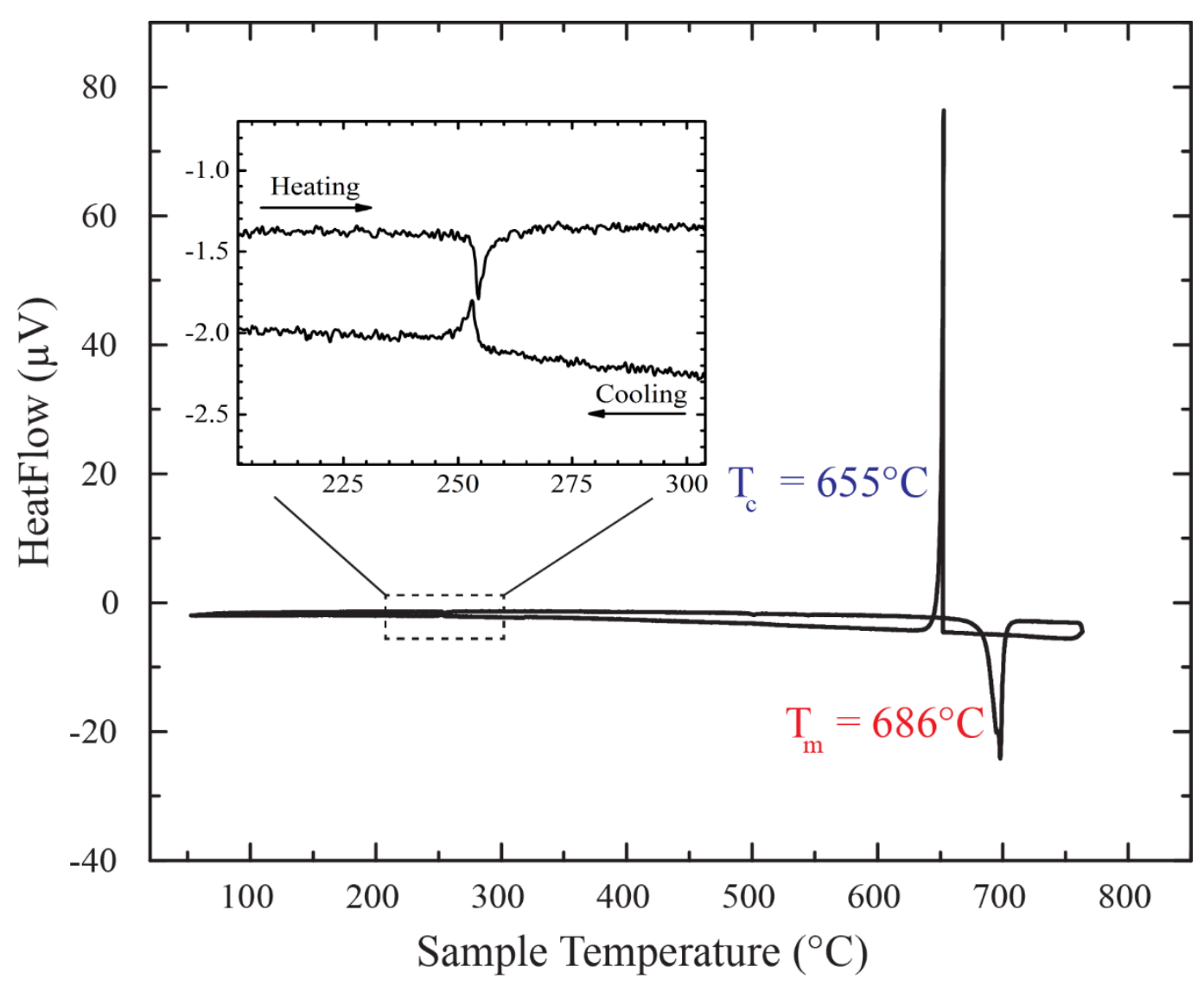

100

Figure 1: The DSC heating and cooling curves for CCI.

During growth in the transparent furnace, the melting point isotherm is typically observed to migrate lower into the cool zone as the ampoule is translated downward due to a heat-sinking effect of the charge which may cause the growth rate to fluctuate over time. In our experience, this effect is minimized by the use of a diaphragm placed between the hot zone and cool zone but is not eliminated, thus some migration of the crystal-melt interface is unavoidable. This results in small variations of the growth rate and interface shape during crystallization.

For CCI grown in quartz ampoules with a wall thickness of $1.5 \mathrm{~mm}$, the interface shape remains approximately flat or slightly convex towards the melt if it can be maintained approximately 1-2 $\mathrm{mm}$ on the hot zone side of a closely fitting diaphragm opening. If the interface is located too far below the diaphragm, (projecting into the cool zone) a slightly concave interface is produced resulting in wall nucleated grains in the boule. This trend is consistent with the theory established by numerical modeling performed by Fu, Chang and Wilcox $[10,11]$.

For each growth experiment, zone settings were tuned to locate the melt/crystal interface at full diameter just above the diaphragm. Prior to beginning translation, all material is remelted leaving a small self-seed a few $\mathrm{mm}$ in height within the bottom of the capillary.

For the $15 \mathrm{~mm}$ diameter growth experiments, the aforementioned process was rather straightforward. Considerable difficulty was encountered in controlling the interface shape 
during the $35 \mathrm{~mm}$ diameter experiment due to the larger cross-sectional area of the interface. A translation rate of $1 \mathrm{~mm} / \mathrm{h}$ was used in all experiments. After all melt had solidified, the crystalline boules were cooled from $\approx 500^{\circ} \mathrm{C}$ to room temperature at constant rates varying from $4 \%$ to $20 \%$ h. Slower rates were used for larger diameters to minimize thermo-elastic stress. After growth and cooling, the ampoules were opened with subsequent cutting and polishing taking place within a nitrogen flushed glove box to prevent decomposition from contact with moisture in the atmosphere. For the purposes of characterization, circular slabs as well as rectangular pixels from each diameter boule were cut using a low speed wire saw with mineral oil as a lubricant.

\subsection{Optical and Scintillation Characterization}

A polariscope coupled with an optical microscope was used to inspect macroscopically observable bulk defects in the grown boules. Contrast was produced through either the Senarmont method or the sensitive color method. Photoluminescence emission and excitation spectra were acquired using a Horiba Fluorolog with a 450W Xenon lamp and a Hamamatsu R928 PMT.

A Hamamatsu 2059 PMT was used for absolute light output measurements under excitation from a ${ }^{137} \mathrm{Cs}$ source on polished samples directly coupled to the PMT window and covered with mineral oil and a diffuse reflector dome made of Spectralon. The manufacturer supplied quantum efficiency for this PMT was convolved with the radioluminescence spectrum of CCI to determine the light yield assuming the light collection efficiency is ideal. Scintillation light yield proportionality was determined as a function of excitation energy using a set of sealed radiation sources with gamma energies ranging from $14 \mathrm{keV}$ to $662 \mathrm{keV}\left({ }^{241} \mathrm{Am},{ }^{57} \mathrm{Co},{ }^{22} \mathrm{Na}\right.$, $\left.{ }^{137} \mathrm{Cs}\right)$. A higher quantum efficiency Hamamatsu R6231-100 PMT was used to acquire pulse height spectra for several volumes of CCI excited by a ${ }^{137} \mathrm{Cs}$ source. Energy resolution was determined from the full width at half maximum (FWHM) of a Gaussian fit of the $662 \mathrm{keV}$ photopeak. Scintillation lifetime was determined under excitation from a Cs-137 source with a $20 \mu$ s shaping time using the time-correlated single photon counting technique [12].

\section{Results and Discussion}

\subsection{Crystal Growth}

The grown boules varied in size with $65 \mathrm{~mm} \mathrm{~L}$ at $\varnothing 15 \mathrm{~mm}, 55 \mathrm{~mm} \mathrm{~L}$ at $\varnothing 22 \mathrm{~mm}$, and $55 \mathrm{~mm} \mathrm{~L}$ at $\varnothing 35 \mathrm{~mm}$. Thermal gradients over $40^{\circ} \mathrm{C} / \mathrm{cm}$ at the diaphragm were prone to cause the crystal to readily crack during growth at $22 \mathrm{~mm}$ and $35 \mathrm{~mm}$ diameters. A reduced thermal gradient of $\approx 20$ $25^{\circ} \mathrm{C} / \mathrm{cm}$ at the diaphragm resulted in preventing severe cracks extending through the entire boule. The formation of transverse cracks occurred more readily for boules with large aspect ratios (length to diameter) at cooling rates above $20^{\circ} \mathrm{C} / \mathrm{h}$. Conversely, boules with aspect ratios closer to 1 did not exhibit any transverse cracking. Boules vary from transparent to slightly cloudy or translucent with a slight yellowish tint under LED light and the bluish emission of the $\mathrm{Eu}^{2+}$ activator in the CCI matrix $(\sim 455 \mathrm{~nm})$ can be observed under fluorescent light. Boules shine a bright blue under ultra-violet light and appear uniform (Figure 2). 


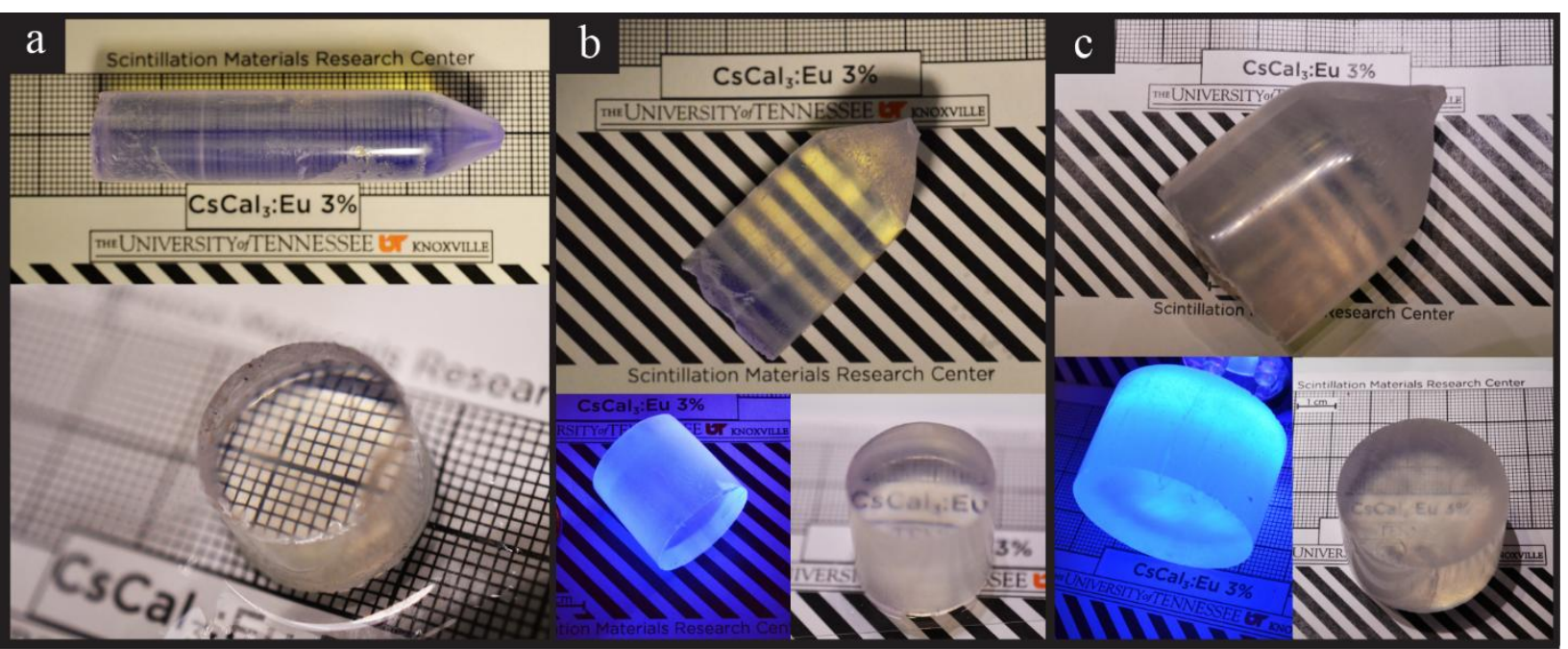

Figure 2: Grown boules of CCI after removal from the ampoule along with polished slabs under fluorescent room light and UV light for $\varnothing 15 \mathrm{~mm}(\mathrm{a}), \varnothing 22 \mathrm{~mm}(\mathrm{~b})$, and $\varnothing 35 \mathrm{~mm}(\mathrm{c})$.

Polished slabs cut from $15 \mathrm{~mm} 22 \mathrm{~mm}$ and $35 \mathrm{~mm}$ boules are clear enough so that fine type can be read through $20 \mathrm{~mm}$ of thickness. Cloudiness in the bulk crystal is more evident in the $22 \mathrm{~mm}$ and $35 \mathrm{~mm}$ diameter boules. Samples of CCI cut easily using a low speed wire saw and can be shaped into rectangular pixels or circular slabs and manually polished using a combination of grinding papers and anhydrous lapping oil.

Towards the end of the crystallization process, a last to freeze region comprising a small fraction of the total volume $(\approx 1-3 \%)$ develops at the top of the charge having a reduced freezing point. It is assumed from experience this forms through segregation of some europium as well as unintentionally added impurities concentrated in this region. Some black impurities thought to be trace carbon also collect here. This impurity laden last to freeze region is typically polycrystalline and is discarded afterwards.

CCI is observed to wet the ampoule forming a concave meniscus with the melt. Separation of the crystal from the ampoule wall upon cooling is indicated by an optical decoupling of the crystal which occurs at temperatures below $400^{\circ} \mathrm{C}$. Following separation from the ampoule wall, vapor species present above the melt charge during and after crystallization first condense and then crystallize on the boule surface as the temperature is decreased. This produces a thin layer of an unidentified secondary phase over most of the exterior which is marked by a significant reduction in transparency looking through the diameter of the ampoule. A time-lapse video documenting the growth and cooling to room temperature of a $\varnothing 22 \mathrm{~mm}$ crystal of CCI using the above equipment is provided along with the electronic version of this text (Link to Video Here).

Rapid decomposition of CCI occurs in normal ambient conditions. Brief exposure to ambient air causes a yellowing of the exterior surface of polished specimens, eventually turning into a white opaque substance from subsequent moisture uptake into the bulk. The yellowing of the surface is believed to be a layer of disassociated iodine caused by decomposition with air. This layer 
greatly impedes light emission from the crystal but can be restored to a pristine condition by removal of the damaged layer by re-polishing. Alternatively, the extreme hygroscopicity of CCI prevents the restoration of scintillation properties if the crystal has adsorbed moisture through deliquescence.

While crystal specimens appear to be single crystals at first approximation, under the polarized light microscope details of the grain macrostructure can be more easily observed (Figure 3). For example, grain boundaries in CCI which appear invisible to the unaided eye can be resolved by the contrast produced by changes in the polarization of the transmitted light. The apparent optical isotropy observed in CCI is in agreement with theoretical calculations predicting a negligible optical anisotropy despite the anisotropic structure [13].

Wall nucleated grains can be observed in the $\varnothing 22 \mathrm{~mm}$ and $\varnothing 35 \mathrm{~mm}$ boules where a concave crystal-melt interface was produced during growth. The polycrystalline grain structure is coarse with grains of a few $\mathrm{mm}$ in diameter. When a flat or convex interface could be achieved, some secondary grains are formed but in general, single grains approaching $10 \mathrm{~mm}$ in diameter are present which is more easily achieved in the $\varnothing 15 \mathrm{~mm}$ boules.

In all samples of CCI investigated, lamellar twinning is observed. One proposed mode of twinning is possible through a solid-solid phase transition from a tetragonal phase at high temperature to the orthorhombic phase observed at room temperature. Evidence of the transition was originally observed through changes to the twin domains when the crystal was heated above $\approx 250^{\circ} \mathrm{C}$ and subsequently cooled after which the original twin domain structures can no longer be observed. A loss in symmetry upon cooling below the suspected transition temperature has been confirmed through high temperature X-ray diffraction studies and will be discussed in detail in a separate manuscript. Similar transitions have been studied for other isostructural perovskite [14] but not specifically for CCI.

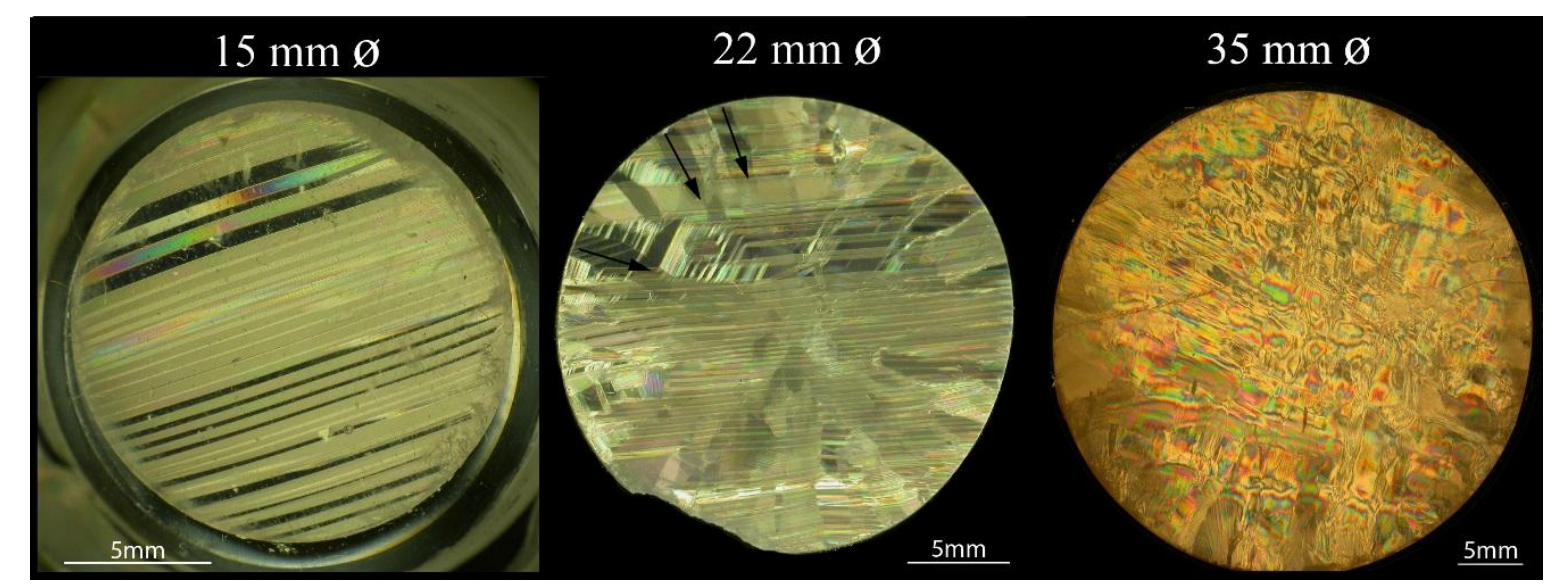

Figure 3: Polarized light microscopy images of CCI grown at $\varnothing 15 \mathrm{~mm}$ exhibiting lamellar twinning of an uncracked singular grain (left) and at $\varnothing 22 \mathrm{~mm}$ the macrostructure is comprised of wall nucleated grains and twinning (right). Arrows are shown depicting the growth of these grains into the bulk. The $\varnothing 35 \mathrm{~mm}$ boule is comprised of heavily strained and twinned grains. 


\subsection{Optical and Scintillation Characterization}

The photoluminescence emission and excitation spectra for CCI at $300 \mathrm{~K}$ is shown in Figure 4. The broad excitation band is seen to overlap the $455 \mathrm{~nm}$ emission of the Eu 5d-4f transition which indicates radiation trapping or self-absorption is likely. These observations are in good agreement with previous reported results from reference [8]. The gamma spectra under excitation from a Cs-137 source of various size samples ranging from $0.1 \mathrm{~cm}^{3}$ to $20 \mathrm{~cm}^{3}$ are shown in Figure 5. It is clear that the asymmetry in the photopeak for larger volume samples indicates inhomogeneity and requires further investigation to determine its nature. It is believed to stem from activator segregation during directional solidification. The difference in the 6 coordinated ionic radius of $\mathrm{Eu}^{2+}$ compared with $\mathrm{Ca}^{2+}$ is large (1.17 $\mathrm{A}$ vs $1.00 \AA$ respectively) [15]. Based upon the ionic radii mismatch, the effective segregation coefficient of $\mathrm{Eu}$ on $\mathrm{Ca}$ sites in $\mathrm{CCI}$ is expected to be $<<1$.

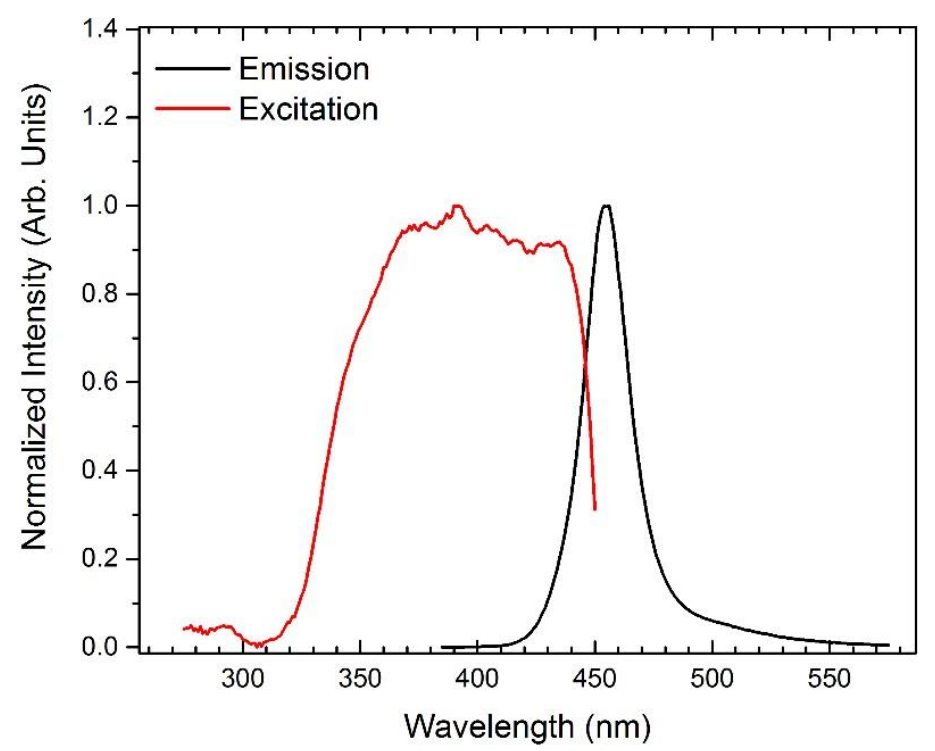

Figure 4. Photoluminescence spectra of CCI. $\lambda_{\mathrm{em}}=465 \mathrm{~nm} \lambda_{\mathrm{ex}}=371 \mathrm{~nm}$.

Due to the peak asymmetry, a fitting of the photopeak using multiple peaks was often required with greater resolution and photopeak symmetry achieved in smaller volume specimens. The energy resolution measured for the $\varnothing 35 \mathrm{~mm}$ x $20 \mathrm{~mm}$ thick slab is approximately $13 \%$ (FWHM/centroid) while an energy resolution for a $2 \times 2 \times 2 \mathrm{~mm}^{3}$ polished cube is approximately $4-4.5 \%$. The improvement in spectroscopic performance is largely attributed to improved volumetric uniformity in the smaller sample as well as the absence of multiple grains of $\mathrm{mm}$ dimension. A slight increase in the measured light yield with decreasing volume can be attributed to improved light collection efficiency as well as fewer scattering sites stemming from twins and grain boundaries. In addition to acting as light scattering sites, grain boundaries are likely locations for impurities to concentrate and are areas of disorder at the atomic scale which would affect charge migration in the crystal. 


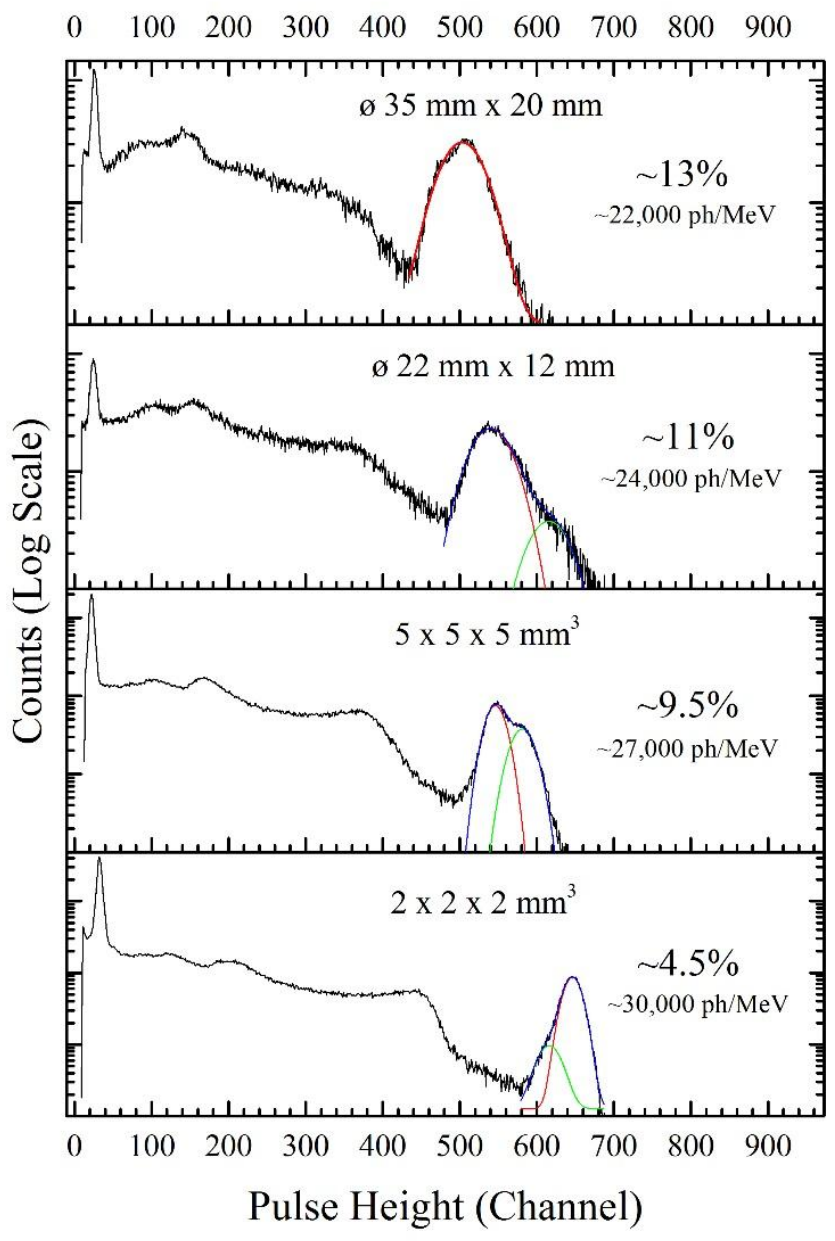

Figure 5: CCI pulse height spectra under excitation by ${ }^{137} \mathrm{Cs}$ gamma source for different sample volumes.

242 The proportionality of light yield (photons/MeV) with photon energy for CCI is shown 243 normalized to the $662 \mathrm{keV}$ response in Figure 6. The sample measured was a $2 \times 2 \times 2 \mathrm{~mm}^{3}$ 244 cleaved specimen containing no visible defects immersed in oil within a quartz cell. Results 245 show very good non-proportionality of less than $5 \%$ at energies as low as $14 \mathrm{keV}$. Error bars 246 provided show variation in repeated measurements. 


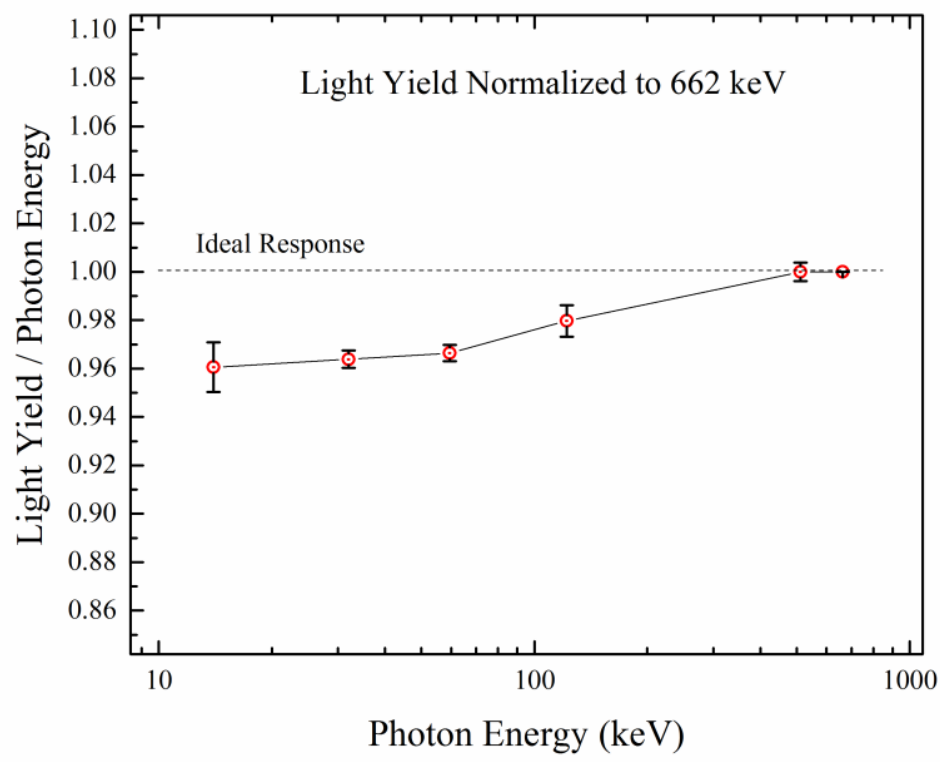

248

249

250

251

252

253

254

255

256

257

258

259

Figure 6: Non-proportionality measurement for CCI.

Scintillation life-time is shown to increase with volume from $\sim 2 \mu$ s (principle component) in a sample measuring $2.5 \times 4 \times 6 \mathrm{~mm}^{3}$ to $\sim 3.8 \mu$ s in a sample measuring $20 \mathrm{~mm} \mathrm{~L}$ × $\varnothing 35 \mathrm{~mm}$ as presented in Figure 7. The lengthening of the scintillation lifetime may be ascribed to the greater bulk defect concentration in the larger crystals but the exact mechanism is unknown at this time. Similar decay time lengthening ascribed to radiation trapping (self-absorption) effects has been observed in other $\mathrm{Eu}^{2+}$ activated compounds as reported in [16]. With regard to pulse height measurements, the longest shaping time used in our investigation was $10 \mu$ s which may limit collection of all scintillation photons for large volume samples with longer decay times and may influence the measured light yield. A summary of scintillation characterization for samples of various volumes of CCI is shown in Table I.

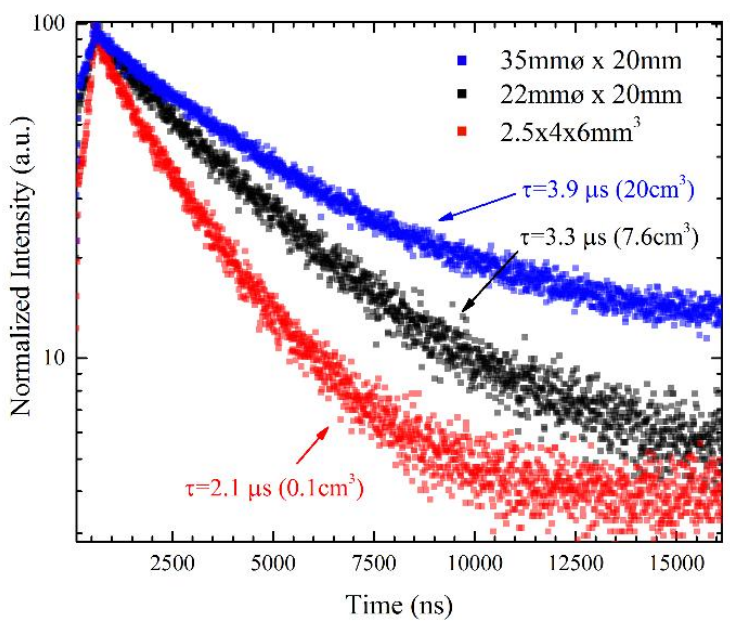


Figure 7: Scintillation decay of CCI for various sample volumes.

Table I. Scintillation Properties of CCI at Various Volumes

\begin{tabular}{lccc}
\hline Sample Size & $\begin{array}{c}\text { Estimated Light Yield } \\
(\mathrm{ph} / \mathrm{MeV})\end{array}$ & $\begin{array}{c}\text { Energy Resolution at 662 } \\
\mathrm{keV}(\mathrm{FWHM} \%)\end{array}$ & $\begin{array}{c}\text { Scintillation } \\
\text { Lifetime } \\
(\mu \mathrm{s})\end{array}$ \\
\hline $2 \times 2 \times 2 \mathrm{~mm}^{3}$ & 30,000 & $\sim 4.5$ & $\mathrm{NA}$ \\
$2.5 \times 4 \times 6 \mathrm{~mm}^{3}$ & $\mathrm{NA}$ & $\mathrm{NA}$ & 2.1 \\
$5 \times 5 \times 5 \mathrm{~mm}^{3}$ & 27,000 & $\sim 9.5$ & $\mathrm{NA}$ \\
$\varnothing 22 \mathrm{~mm} \times 12 \mathrm{~mm}$ & 24,000 & $\sim 11$ & $\mathrm{NA}$ \\
$\varnothing 22 \mathrm{~mm} \times 20 \mathrm{~mm}$ & $\mathrm{NA}$ & $\mathrm{NA}$ & 3.3 \\
$\varnothing 35 \mathrm{~mm} \times 20 \mathrm{~mm}$ & 22,000 & $\sim 13$ & 3.9 \\
\hline
\end{tabular}

We have demonstrated the growth of crystals of CCI using the vertical Bridgman technique with improved energy resolution and optical quality over previously reported results. The measured energy resolution for a single crystal approaches 4\% (FWHM) at $662 \mathrm{keV}$ which is a large improvement over early estimates of $8 \%$ [8] and $16 \%$ as reported in [7]. Single crystals isolated from polycrystalline boules present promising performance with both energy resolution and proportionality that surpasses that of NaI:Tl. With further optimization of the growth process, drawback encountered in CCI is the demonstrated non-uniformity which may be due to inhomogeneous activator distribution as other investigators have pointed out. To confirm the segregation behavior of the activator, an analysis of the Eu concentration changes across the volume of the crystal is required. Moreover, the primary challenges to the further development of CCI lay in improving uniformity in larger volume crystals and prevention of secondary grain growth as well as twinning.

278

\section{References}

[1] P. Lecoq, A. Annenkov, A. Gektin, M. Korzhik, C. Pedrini, Inorganic Scintillators for Detector Systems: Physical Principles and Crystal Engineering, in: A. Annenkov, A. Gektin, M. Korzhik, C. Pedrini (Eds.), Springer, Berlin, Heidelberg, 2006, pp. 124-173.

[2] R. Hawrami, J. Glodo, K.S. Shah, N. Cherepy, S. Payne, A. Burger, L. Boatner, Bridgman bulk growth and scintillation measurements of SrI2:Eu2+, Journal of Crystal Growth, 379 (2013) 69-72.

[3] W.M. Higgins, A. Churilov, E. van Loef, J. Glodo, M. Squillante, K. Shah, Crystal growth of large diameter LaBr3:Ce and CeBr3, Journal of Crystal Growth, 310 (2008) 2085-2089.

[4] E.D. Bourret-Courchesne, G.A. Bizarri, R. Borade, G. Gundiah, E.C. Samulon, Z. Yan, S.E. Derenzo, Crystal growth and characterization of alkali-earth halide scintillators, Journal of Crystal Growth, 352 (2012) 78-83. 
[5] M. Zhuravleva, C.L. Melcher, L. Stand, A. Lindsey, H. Wei, C. Hobbs, M. Koschan, High energy resolution scintillators for nuclear nonproliferation applications, in, 2014, pp. 921303-921303-921312.

[6] A.Y. Grippa, N.V. Rebrova, T.E. Gorbacheva, V.Y. Pedash, N.N. Kosinov, V.L. Cherginets, V.A. Tarasov, O.A. Tarasenko, A.V. Lopin, Crystal growth and scintillation properties of $\mathrm{CsCaBr} 3: \mathrm{Eu} 2+(\mathrm{CsCa} 1-\mathrm{xEuxBr} 3,0 \leq \mathrm{x} \leq 0.08)$, Journal of Crystal Growth, 371 (2013) 112-116.

[7] E. Rowe, E. Tupitsyn, B. Wiggins, P. Bhattacharya, L. Matei, M. Groza, V. Buliga, A. Burger, P. Beck, N.J. Cherepy, S.A. Payne, Double Salts Iodide Scintillators: Cesium Barium Iodide, Cesium Calcium Iodide, and Barium Bromine Iodide, Crystal Research and Technology, 48 (2013) 227-235.

[8] M. Zhuravleva, B. Blalock, K. Yang, M. Koschan, C.L. Melcher, New single crystal scintillators: CsCaCl3:Eu and CsCaI3:Eu, Journal of Crystal Growth, 352 (2012) 115-119.

[9] A.C. Lindsey, M. Zhuravleva, C.L. Melcher, Growth of $\mathrm{CsCe} 2 \mathrm{Cl} 7$ and $\mathrm{Cs} 3 \mathrm{CeCl6}$ utilizing the Bridgman method, in: Nuclear Science Symposium and Medical Imaging Conference (NSS/MIC), 2013 IEEE, 2013, pp. 1-5.

[10] C.E. Chang, W.R. Wilcox, Control of Interface Shape in Vertical Bridgman-Stockbarger Technique, Journal of Crystal Growth, 21 (1974) 135-140.

[11] T.W. Fu, W.R. Wilcox, Influence of Insulation on Stability of Interface Shape and Position in the Vertical Bridgman-Stockbarger Technique, Journal of Crystal Growth, 48 (1980) 416424.

[12] L.M. Bollinger, G.E. Thomas, Measurement of the Time Dependence of Scintillation Intensity by a Delayed-Coincidence Method, Rev. Sci. Instrum., 32 (1961) 1044-1050.

[13] M. Tyagi, M. Zhuravleva, C.L. Melcher, Theoretical and experimental characterization of promising new scintillators: $\mathrm{Eu} 2+$ doped $\mathrm{CsCaCl} 3$ and $\mathrm{CsCaI} 3$, Journal of Applied Physics, 113 (2013) -.

[14] S.A. Redfern, High-temperature structural phase transitions in perovskite, Journal of Physics: Condensed Matter, 8 (1996) 8267.

[15] R. Shannon, Revised effective ionic radii and systematic studies of interatomic distances in halides and chalcogenides, Acta Crystallogr. Sect. A, 32 (1976) 751-767.

[16] J. Glodo, E. van Loef, R. Hawrami, U. Shirwadkar, S. Mukhopadhyay, K.S. Shah, Ieee, Spectroscopy of Selected Alkaline Earth Halides, 2010 Ieee Nuclear Science Symposium Conference Record (Nss/Mic), (2010) 271-274.

\section{Acknowledgment}

This work has been supported by the US Department of Homeland Security, Domestic Nuclear Detection Office, under competitively awarded grant \#2012-DN-077-ARI067-04. The high temperature X-ray diffraction experiments were performed using the instruments that were procured through the general infrastructure grant of DOE-Nuclear Energy University Program (DE-NE0000693). This support does not constitute an express or implied endorsement on the part of the Government. We are also grateful for the generous help of Dr. Maulik Patel for his assistance in preparing this manuscript. 